$6-1-2004$

\title{
Synchronous Motor Phase Control By Vector Addition of Induced Winding Voltages
}

\author{
Daniel J. Simon \\ Cleveland State University, d.j.simon@csuohio.edu \\ Dennis L. Feucht \\ Innovatia Laboratories, dennis@innovatia.com
}

Follow this and additional works at: https://engagedscholarship.csuohio.edu/enece_facpub

Part of the Electrical and Computer Engineering Commons, and the Structural Materials Commons How does access to this work benefit you? Let us know!

\section{Publisher's Statement}

(C)2004 IEEE. Personal use of this material is permitted. Permission from IEEE must be obtained for all other users, including reprinting/ republishing this material for advertising or promotional purposes, creating new collective works for resale or redistribution to servers or lists, or reuse of any copyrighted components of this work in other works.

\section{Original Citation}

Simon, D.; Feucht, D.L.; , "Synchronous motor phase control by vector addition of induced winding voltages," Industrial Electronics, IEEE Transactions on , vol.51, no.3, pp. 537- 544, June 2004 doi: 10.1109/ TIE.2004.825367

\section{Repository Citation}

Simon, Daniel J. and Feucht, Dennis L., "Synchronous Motor Phase Control By Vector Addition of Induced Winding Voltages" (2004). Electrical Engineering \& Computer Science Faculty Publications. 21.

https://engagedscholarship.csuohio.edu/enece_facpub/21

This Article is brought to you for free and open access by the Electrical Engineering \& Computer Science Department at EngagedScholarship@CSU. It has been accepted for inclusion in Electrical Engineering \& Computer Science Faculty Publications by an authorized administrator of EngagedScholarship@CSU. For more information, please contact library.es@csuohio.edu. 


\title{
SYNCHRONOUS MOTOR PHASE CONTROL BY VECTOR ADDITION OF INDUCED WINDING VOLTAGES
}

\author{
Dan Simon, Cleveland State University
}

\author{
Dennis L. Feucht
}

\begin{abstract}
We propose a simple low-cost approach to the winding-sensed control of three-phase synchronous permanent-magnet motors. Our approach results from a straightforward but elegant application of vector-based motor theory. The method therefore does not depend on the differential equations that describe the motor dynamics, and it does not depend on the particular motor parameters. The single method we derive applies to both wye- and delta-configured motors. We vectorially sum the voltage waveforms at the motor terminals in a certain way and use their zero crossings to produce a correctly phased six-step switch sequence. We discuss the implementation for both wyeand delta-configured motors, and we present experimental results for a delta-configured implementation.
\end{abstract}

Index Terms-Brushless rotating machines, motor drives, permanent-magnet motors.

\section{INTRODUCTION}

$\mathbf{T}$ HE permanent-magnet synchronous (PMS) motors, also called "brushless dc motors," have been applied extensively over the past few decades. Their applications include compact disk players, disk drives, robotic manufacturing, air conditioning, and artificial hearts [8]. PMS motors are popular partly because of their high power density, high torque-to-inertia ratio, high efficiency, and relative ease of control [2]. However, sensing rotor position (using Hall sensors, encoders, or resolvers) for closed-loop control constrains the use of PMS motors, reducing reliability and increasing complexity.

Various methods have been proposed for winding-sensed control of PMS motors. We restrict discussion here to induced voltage sensing to sense rotor position. For maximum torque for a given winding current, switch sequence step changes must occur at zero crossings $30^{\circ}$ out of phase with those of the terminal waveforms, which occur midway between switch sequence steps. The problem has been addressed by low-pass filters (to provide phase delays of the induced voltages) [2], digital phase shifters [6], phase-locked loops (to estimate the zero-crossings of the induced voltages [12]), comparison of the induced voltage integrals with threshold voltages [1], and the use of line-referenced induced voltages (which lead the phase-referenced induced voltages by $30^{\circ}$ ) [2].

Other methods are based on sensed motor currents [5], fluxlinkage estimation [11], or injection of high-frequency voltages into the motor windings [4]. Some researchers have proposed

D. Simon is with the Electrical Engineering Department, Cleveland State University, Cleveland, OH 44115 USA (e-mail: d.j.simon@ @suohio.edu).

D. L. Feucht can be reached via General Delivery, San Ignacio, Cayo, Belize.

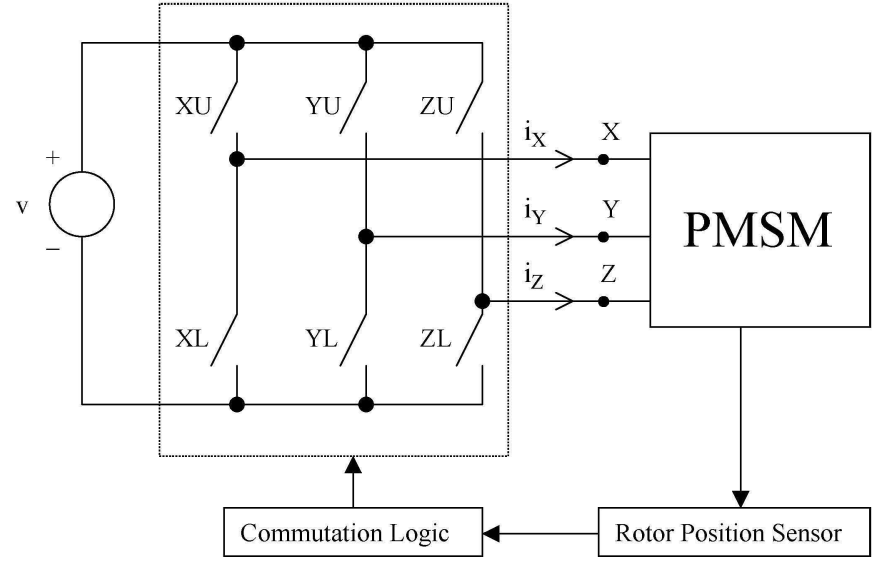

Fig. 1. Overview of motor drive.

a combination of current and flux linkage estimation [9] or methods based on magnetic saliency [10]. State-space methods include linear state-space observers [15], nonlinear observers [14], sliding-mode observers [3], Kalman filters [13], fuzzy logic [7], and neural network observers [16].

The motor-drive scheme assumed here is shown in Fig. 1. The wye- or delta-configured motor has three terminals labeled $X, Y$, and $Z$ with currents $i_{X}, i_{Y}$, and $i_{Z}$. Two switches are closed at any one time. The switch sequence determines when the various switches open and close. In general, the switching sequence is periodic. This paper presents a method for acquiring rotor position by a straightforward but elegant application of vector-based motor theory. This method does not depend on the differential equations that describe the motor dynamics, and it does not depend on the particular motor parameters. The method applies to both three-phase winding configurations. Our approach is to vectorially sum the voltage waveforms at the motor terminals in a certain way and use their zero crossings to produce a correctly phased six-step switch sequence. Section II explains the method using the wye configuration. Section III presents vector-summed control. Section IV discusses implementation details in wye-configured motors, and Section $\mathrm{V}$ extends it to the delta-configured case. Section VI presents our experimental results, and Section VII contains concluding remarks and discussion.

\section{SiX-STEP Phase CONTROL}

Fig. 2 shows the equivalent circuit model for a wye-configured motor during the $\mathrm{YZ}$ drive step. The currents into the three terminals are labeled $i_{X}, i_{Y}$, and $i_{Z}$. The currents in each phase winding are labeled $i_{A}, i_{B}$, and $i_{C}$. For wye-configured motors 


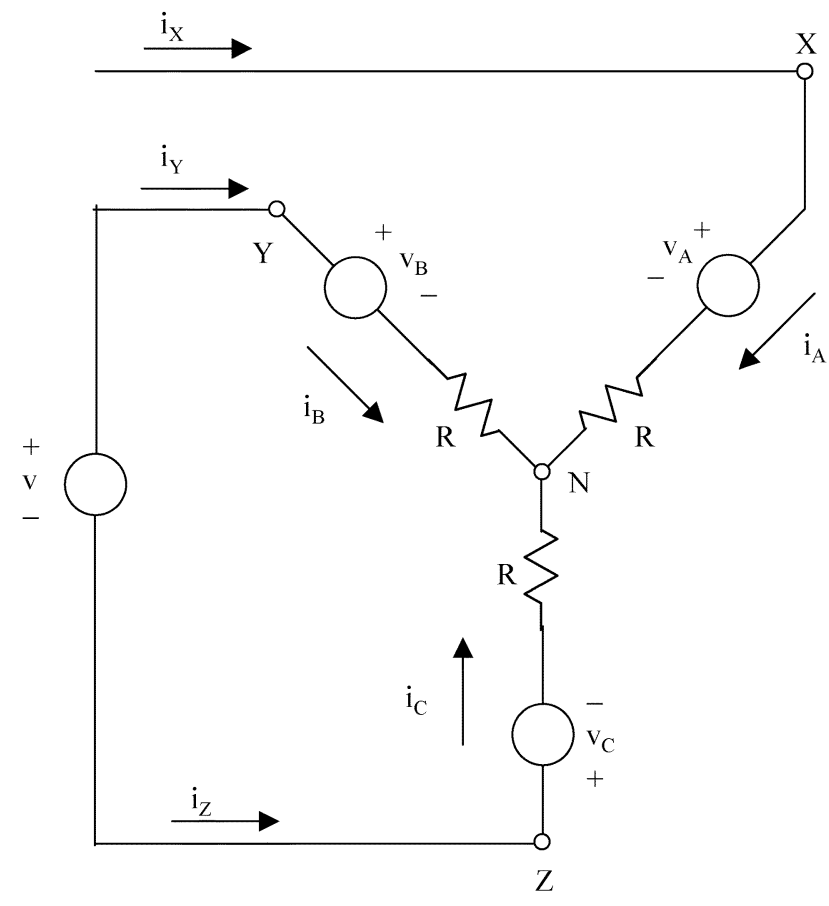

Fig. 2. Circuit model of a three-phase wye-configured motor.

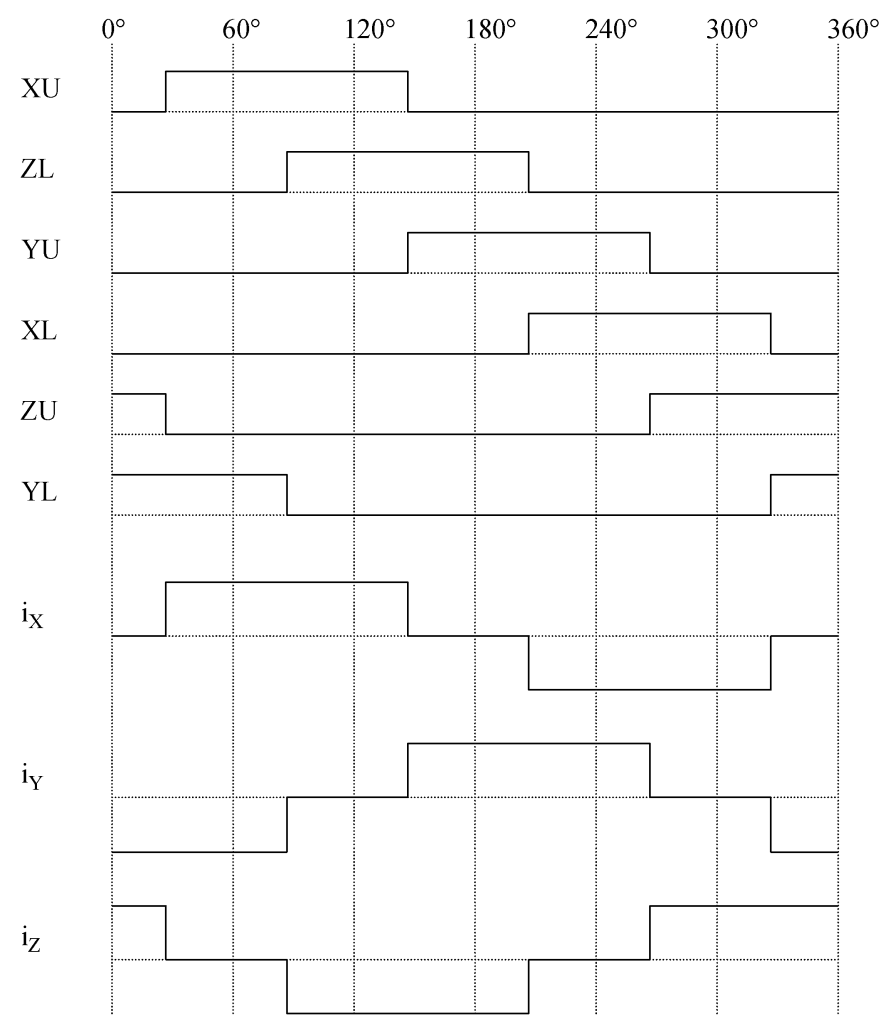

Fig. 3. Switching logic and terminal currents for a three-phase PMS motor.

we see that $i_{A}=i_{X}, i_{B}=i_{Y}$, and $i_{C}=i_{Z}$. The voltage sources are the induced voltages of each phase-winding, induced by the rotor. Fig. 3 shows one period of the switch sequence and the corresponding phase-winding currents. It can be seen that from $30^{\circ}$ to $90^{\circ}$, switches $\mathrm{XU}$ and $\mathrm{YL}$ are on. Therefore, positive current flows through winding $\mathrm{A}$, negative current through winding $\mathrm{B}$, with no current through winding $\mathrm{C}$.
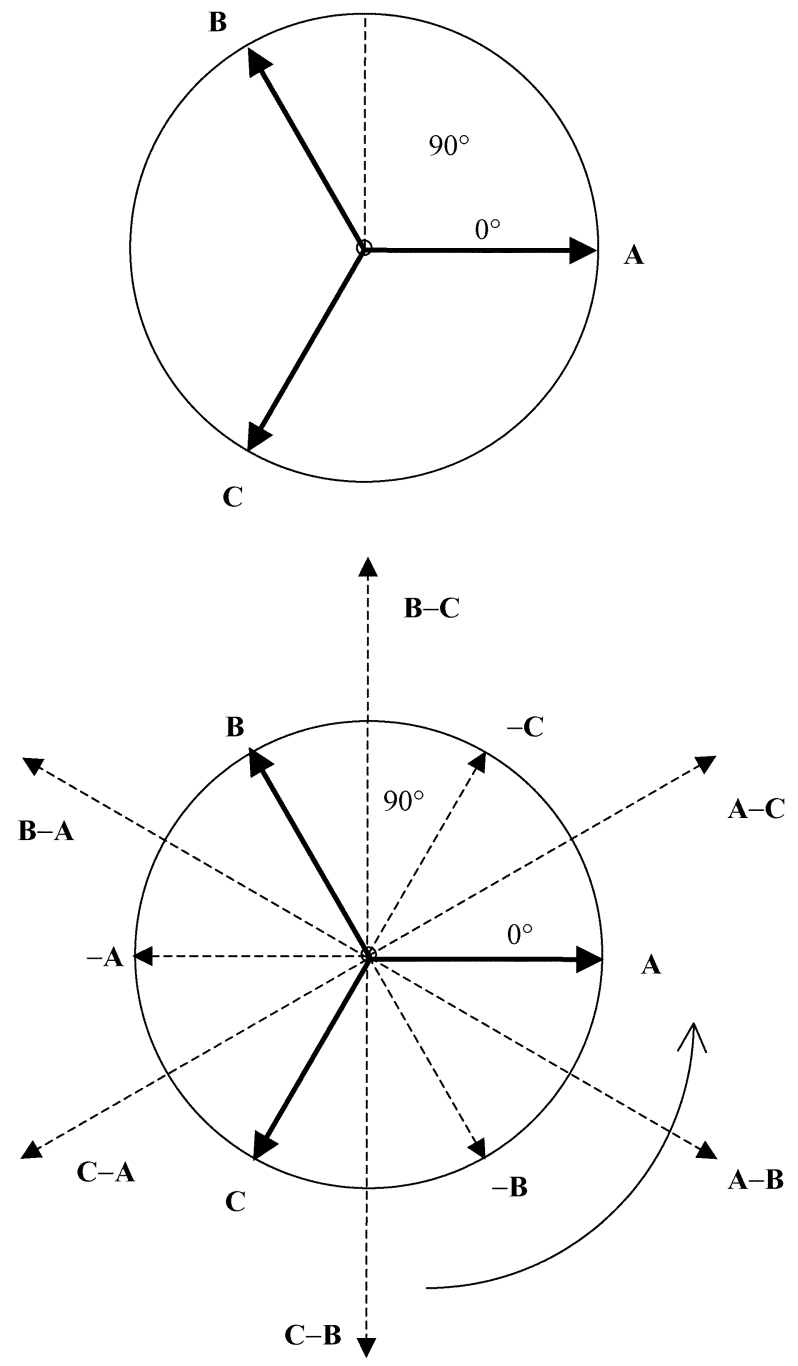

Fig. 4. Winding arrangement and stator field differences for a three-phase motor.

With $120^{\circ}$ phase separation between windings, as shown in Fig. 4, between $30^{\circ}-90^{\circ}$ of the period (when switches XU and $\mathrm{YL}$ are on) the stator field vector will be pointed in the direction of $\mathbf{A}-\mathbf{B}$ as shown in Fig. 4(b). From $90^{\circ}$ to $150^{\circ}$ (when switches $\mathrm{XU}$ and $\mathrm{ZL}$ are on) positive current flows through winding $A$ and negative current flows through winding C. The stator field will then be pointed in the direction of the A-C vector, as shown. As the switching proceeds through one complete period, the stator field rotates in a counterclockwise $(\mathrm{CCW})$ direction around the stator, in $60^{\circ}$ steps.

As the stator field rotates, the rotor follows it. For maximum motor torque, the rotor lags the stator field by $90^{\circ}$, but cannot maintain constant $90^{\circ}$ lag because the orientation of the stator field is limited to $60^{\circ}$ resolution. This scheme reduces the phase error to $\pm 30^{\circ}$. For example, the $\mathbf{A}-\mathbf{B}$ field is at $-30^{\circ}$ in Fig. 4(b). Consequently, we should turn on $\mathbf{A}-\mathbf{B}$ when the rotor reaches $-150^{\circ}$ and turn it off when the rotor reaches $-90^{\circ}$. Likewise, we should turn on the $\mathbf{A}-\mathbf{C}$ field when the rotor reaches $-90^{\circ}$ and turn it off when the rotor reaches $-30^{\circ}$. This results in torque ripple in the motor, but the torque variation is only $\left(1-\cos 30^{\circ}\right)=13.4 \%$, which is acceptable for many low-cost control applications. As the permanent-magnet rotor 


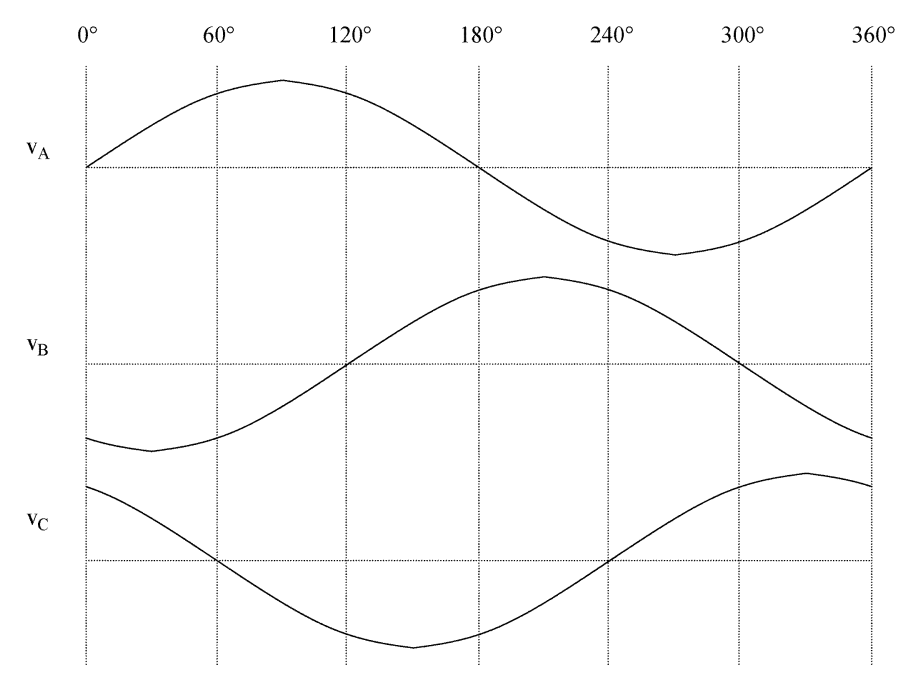

Fig. 5. Induced winding voltages for a wye-configured motor.

TABLE I

SWITCHING LOGIC FOR Wye-CONFIGURED Motor BASED ON INDUCED VOLTAGES

\begin{tabular}{c|c|r}
\hline \hline Drive Step & Switches to Turn On & When to Turn Switches On \\
\hline XY & YL and XU & $30^{\circ}$ after + zero crossing of A \\
XZ & XU and ZL & $210^{\circ}$ after + zero crossing of C \\
YZ & ZL and YU & $30^{\circ}$ after + zero crossing of $\mathbf{B}$ \\
YX & YU and XL & $210^{\circ}$ after + zero crossing of A \\
ZX & XL and ZU & $30^{\circ}$ after + zero crossing of C \\
ZY & ZU and YL & $210^{\circ}$ after + zero crossing of B \\
\hline \hline
\end{tabular}

rotates, it induces voltages in the phase windings. If the motor is running at maximum torque as described above, then the induced voltages in the three phases will appear as sine waves as shown in Fig. 5. The induced voltage in phase B lags that in phase $\mathrm{A}$ by $120^{\circ}$, and the induced voltage in phase $\mathrm{C}$ lags that in phase $\mathrm{B}$ by $120^{\circ}$.

For winding-sensed control, we want to use the induced voltage zero crossings to determine the switching points of the switches in Fig. 1. Comparison of Figs. 3 and 5 show that the zero crossings occur exactly halfway between the desired switching points. Therefore, the induced voltages need to be processed somehow to determine the desired switching points.

\section{VECTOR-SUMMED CONTROL}

From Figs. 3 and 5, we see that we want the XU/YL drive step to begin $30^{\circ}$ after the positive zero-crossing of $v_{A}$. We will refer to the $\mathrm{XU} / \mathrm{YL}$ drive step as the $\mathrm{XY}$ drive step since the positive terminal of the voltage source is connected to node $X$ and the ground is connected to node Y. We next want the XU/ZL drive step to begin at $90^{\circ}$, which is $30^{\circ}$ after the negative zero crossing of $v_{C}$ (this will generate an $\mathrm{XZ}$ drive step). The entire switch sequence is shown in Table I.

A comparison of Figs. 3 and 5 shows that both zero crossings of the induced voltage in a given phase-winding occurs when that phase winding does not have any current. For instance, from Fig. 5 we see that the zero crossings of $v_{A}$ in phase A occur at $0^{\circ}$ and $180^{\circ}$. From Fig. 3 we see that $i_{X}$ is zero at $0^{\circ}$ and $180^{\circ}$. Similar observations can be made for $v_{B}$ and $v_{C}$. Therefore, if we can synthesize a sinusoid that lags $v_{A}$ by $30^{\circ}$ then we can use

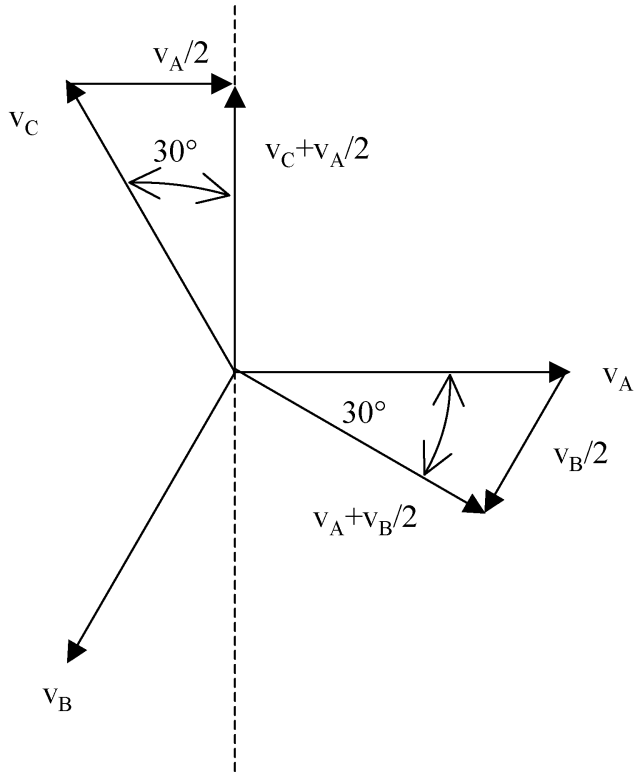

Fig. 6. Induced voltage vector addition.

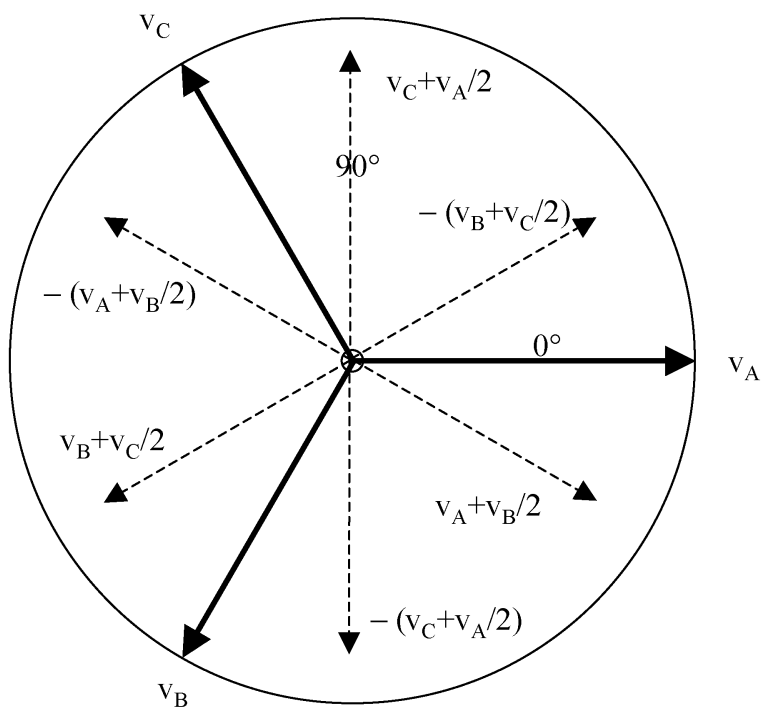

Fig. 7. Vector diagram of induced winding voltages for a wye-configured motor.

its positive zero crossing to turn on the $\mathrm{XY}$ drive step. Likewise, a sinusoid that lags $v_{C}$ by $210^{\circ}$ can use its positive zero crossing to turn on the XZ drive step. Similar statements can be made for the other drive steps.

A vector with a $30^{\circ}$ offset can be synthesized from terminal voltages as shown in Fig. 6 . The induced voltage sum $\left(v_{A}+\right.$ $\left.v_{B} / 2\right)$ lags $v_{A}$ by $30^{\circ}$. Similarly, note that the sum $\left(v_{C}+v_{A} / 2\right)$ lags $v_{C}$ by $30^{\circ}$. This procedure can be continued to obtain six voltage sums with zero crossings that lag the induced voltage zero crossings by various angles. These relationships are shown in Fig. 7. Therefore, if we can synthesize $\left(v_{A}+v_{B} / 2\right)$ then we can use its positive zero crossing to turn on the $\mathrm{XY}$ drive step. Note from Fig. 7 that the XY drive step is turned on when the induced voltage across the XY terminals has a phase of $60^{\circ}$, which is the same time at which the vector-summed voltage $\left(v_{A}+v_{B} / 2\right)$ crosses zero in the positive direction. Likewise, 
TABLE II

SWITCHING LOGIC FOR WYE-CONFIGURED MOTOR BASED ON INDUCED VOLTAGE SUMS

\begin{tabular}{c|c|l}
\hline \hline Drive Step & Switches to Turn On & When to Turn Switches On \\
\hline $\mathrm{XY}$ & YL and XU & + zero crossing of $v_{A}+v_{B} / 2$ \\
$\mathrm{XZ}$ & $\mathrm{XU}$ and ZL & - zero crossing of $v_{C}+v_{A} / 2$ \\
$\mathrm{YZ}$ & ZL and YU & + zero crossing of $v_{B}+v_{C} / 2$ \\
$\mathrm{YX}$ & $\mathrm{YU}$ and XL & - zero crossing of $v_{A}+v_{B} / 2$ \\
$\mathrm{ZX}$ & $\mathrm{XL}$ and $\mathrm{ZU}$ & + zero crossing of $v_{C}+v_{A} / 2$ \\
$\mathrm{ZY}$ & $\mathrm{ZU}$ and $\mathrm{YL}$ & - zero crossing of $v_{B}+v_{C} / 2$ \\
\hline \hline
\end{tabular}

if we can synthesize $\left(v_{C}+v_{A} / 2\right)$ then we can use its negative zero crossing to turn on the XZ drive step. Note from Fig. 7 that the $X Z$ drive step is turned on when the induced voltage across the XZ terminals has a phase of $60^{\circ}$, which is the same time at which the vector-summed voltage $\left(v_{C}+v_{A} / 2\right)$ crosses zero in the negative direction. Similar statements can be made about the other drive steps. Therefore, a switch sequence table equivalent to Table I can be formulated as shown in Table II.

\section{WYE IMPLEMENTATION}

Now, consider the case during the $\mathrm{YZ}$ drive step when we want to use the negative zero crossing of $\left(v_{A}+v_{B} / 2\right)$ to turn on the YX drive step. Then, the motor is operating between $150^{\circ}-210^{\circ}$ in Fig. 3. Therefore, switches YU and ZL are on, phase A has no current, phase $\mathrm{B}$ has positive current, and phase $\mathrm{C}$ has negative current. Then, referring to Fig. 2, $v_{A}, v_{B}$, and $v_{C}$ cannot be sensed directly, but the terminal voltages $v_{X}, v_{Y}$, and $v_{Z}$ are available. From Fig. 2,

$$
\begin{aligned}
& v_{N}=-v_{B}-i R+v \\
& v_{N}=-v_{C}+i R .
\end{aligned}
$$

Adding these equations and recognizing that $v_{A}+v_{B}+v_{C}=0$ (see Figs. 5 and 7),

$$
v_{N}=\frac{\left(v_{A}+v\right)}{2}
$$

Fig. 2 shows that the terminal voltage $v_{X}$ can be written as

$$
v_{X}=v_{A}+v_{N}
$$

If the voltage at node $N$ was available for measurement, we could compute $v_{A}$ from this equation by subtracting $v_{N}$ from $v_{X}$. However, to reduce motor manufacturing costs, node $N$ is not usually available outside the motor. We therefore combine the two preceding equations to obtain

$$
v_{X}=\frac{3}{2} v_{A}+\frac{1}{2} v
$$

Next, we connect three equal-valued resistors (with large values) to the three phase terminals as shown in Fig. 8. We can use KCL at node $N_{V}$ to derive

$$
3 v_{N V}=v_{X}+v_{Y}+v_{Z}
$$

Recognizing from Fig. 8 that $v_{Y}=v$ and $v_{Z}=0$, we obtain

$$
v_{N V}=\frac{\left(v_{X}+v\right)}{3}
$$

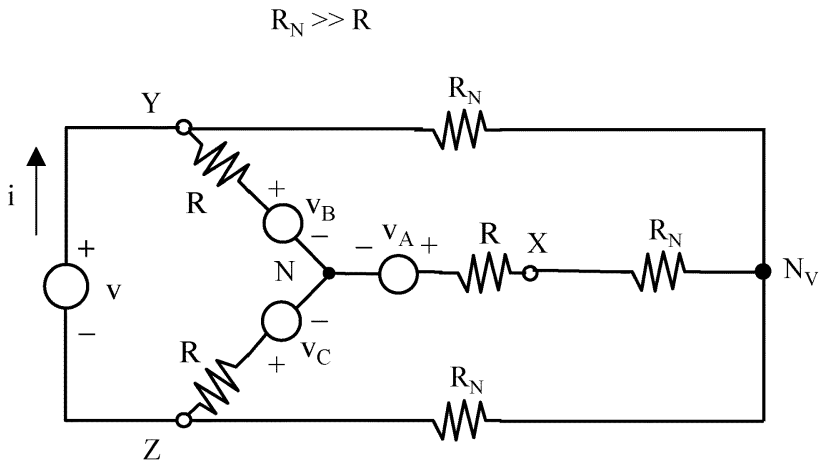

Fig. 8. Circuit model of a wye-configured motor with a virtual neutral node during the $\mathrm{YZ}$ drive step.

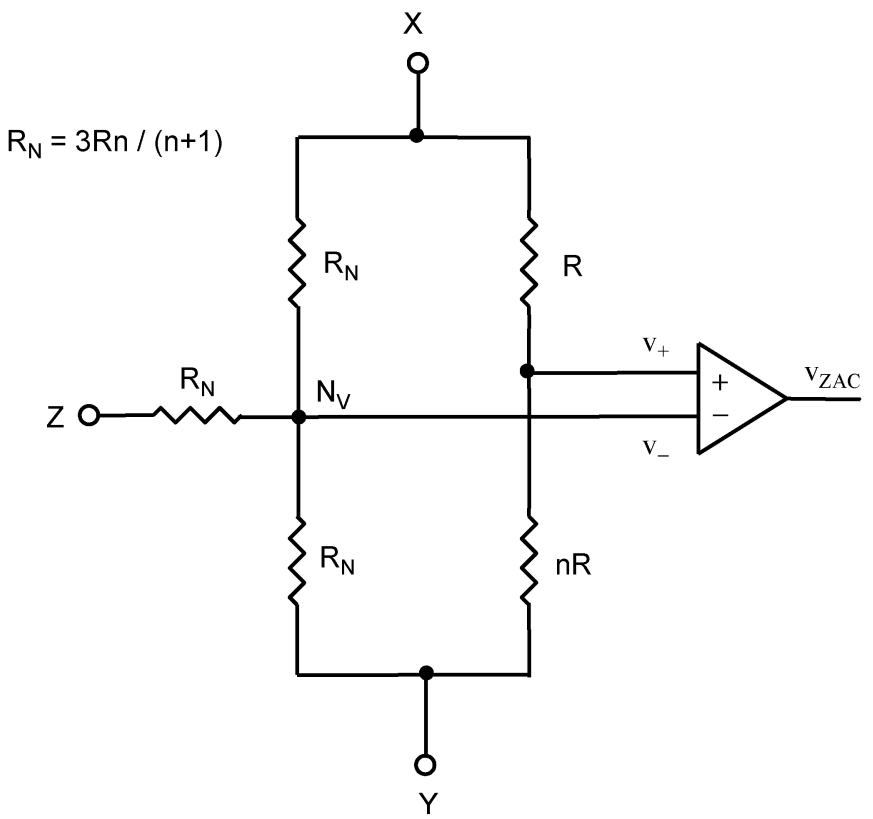

Fig. 9. Phase-summing implementation during the YZ drive step.

Combining this equation and (5) to eliminate $v$ results in

$$
v_{A}=v_{X}-v_{N V} \text {. }
$$

This shows that when node $\mathrm{X}$ is open, $v_{A}$ is equal to the difference between $v_{X}$ and $v_{N V}$. Similar procedures are used to sense induced voltages $v_{B}$ and $v_{C}$ when phases $\mathrm{B}$ and $\mathrm{C}$ are undriven.

Next, we add the circuitry shown in Fig. 9 to terminals $\mathrm{X}, \mathrm{Y}$, and $N_{V}$, where $n$ in the figure is a resistance ratio to be determined. It can be derived from Fig. 9 that

$$
\begin{aligned}
v_{+} & =\frac{n}{n+1} v_{X}+\frac{1}{n+1} v_{Y} \\
& =\frac{n}{n+1}\left(v_{X}+\frac{1}{n} v\right)
\end{aligned}
$$

where the second equality follows from the drive constraint, $v_{Y}=v$. From Fig. 9 and (7),

$$
\begin{aligned}
v_{-} & =v_{N V} \\
& =\frac{\left(v_{X}+v\right)}{3} .
\end{aligned}
$$


Substituting (5) for $v_{X}$ results in

$$
v_{-}=\frac{\left(v_{A}+v\right)}{2} \text {. }
$$

Substituting (5) for $v_{X}$ in (10) gives

$$
\begin{aligned}
v_{+} & =\frac{n}{n+1}\left(\frac{3 v_{A}}{2}+\frac{v}{2}+\frac{v}{n}\right) \\
& =\frac{3 n v_{A}}{2(n+1)}+\frac{(n+2) v}{2(n+1)} .
\end{aligned}
$$

Combining this with (13) gives the total comparator input voltage as

$$
v_{+}-v_{-}=\frac{2 n-1}{2(n+1)} v_{A}+\frac{1}{2(n+1)} v .
$$

From motor symmetry, $v_{A}, v_{B}$, and $v_{C}$ have the same amplitudes (as shown in Figs. 5 and 7), $V$. Vector addition in Fig. 7 results in voltage differences having a magnitude equal to $\sqrt{3}$ times the individual phase-winding amplitudes. From Fig. 7, $v_{B}$ is $90^{\circ}$ ahead of $v_{C A} \equiv\left(v_{C}-v_{A}\right)$. Therefore, $v_{B}$ is maximum when $v_{C A}=0$. We can further see from Fig. 7 that $v_{B C}$ (which has the same phase as $\left.-\left(v_{C}+v_{A} / 2\right)\right)$ leads $v_{C A}$ by $120^{\circ}$. Therefore, when $v_{C A}$ is at its positive zero crossing,

$$
\begin{aligned}
v_{B C} & =V_{B C} \sin \left(120^{\circ}\right) \\
& =\sqrt{3} V\left(\frac{\sqrt{3}}{2}\right) \\
& =\frac{3 V}{2} .
\end{aligned}
$$

During the YZ drive step, it can be seen from Fig. 2 that $v_{Y Z}=$ $v$. At the maximum speed, $v_{B C}=v_{Y Z}$ during the $\mathrm{YZ}$ drive step, and (19) can be written as

$$
v=\frac{3 V}{2} .
$$

Recall from Table II that we can use the negative zero crossing of $\left(v_{A}+v_{B} / 2\right)$ (which occurs at the same time as the positive zero crossing of $\left.\left(v_{C}-v_{A}\right)\right)$ to begin the YX drive step, which follows the YZ drive step. $\left(v_{A}+v_{B} / 2\right)$ is not directly available, but can be synthesized as the comparator input voltage difference $\left(v_{+}-v_{-}\right)$, shown in Fig. 9. At the positive zero crossing of $\left(v_{C}-v_{A}\right)$ we know that $v_{B}$ is at its maximum magnitude $(V)$ as discussed above. Therefore, (20) tells us that at the positive zero crossing of $\left(v_{C}-v_{A}\right)$ we have $v=3 v_{B} / 2$. Therefore, at the positive zero crossing of $v_{C A}$, (16) can be written as

$$
v_{+}-v_{-}=\frac{2 n-1}{2(n+1)} v_{A}+\frac{1}{2(n+1)} \frac{3 v_{B}}{2} .
$$

For $n=2$ this is equal to $\left(v_{A}+v_{B} / 2\right) / 2$. Therefore, if we use the resistance ratio $n=2$ in Fig. 9, the negative zero crossing of $\left(v_{+}-v_{-}\right)$can be used to begin the YX drive step.

This reasoning can be extended to construct the comparator circuits for the complete switching sequence, as shown in Fig. 10, with sequencing shown in Table III.

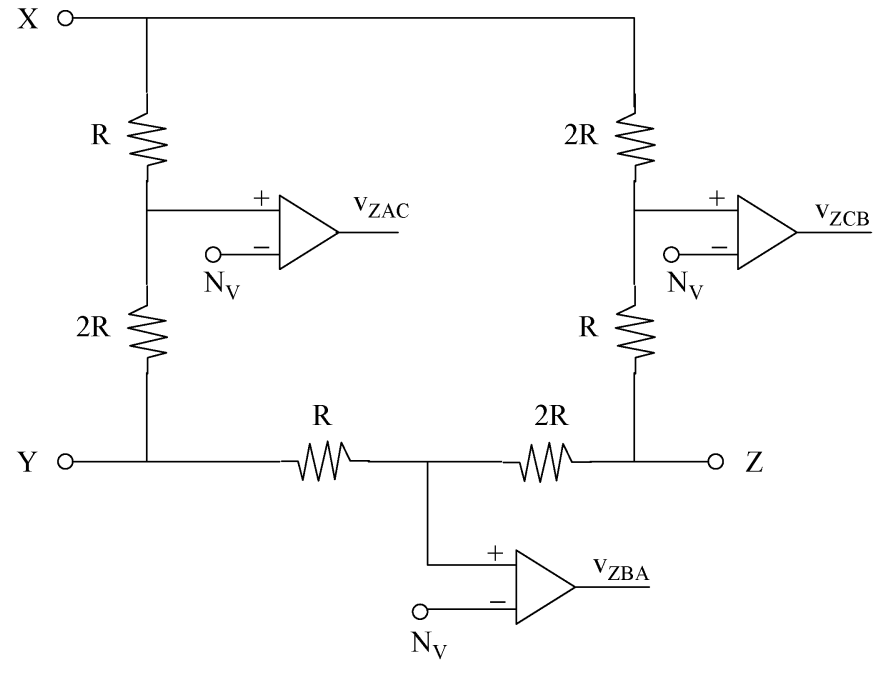

Fig. 10. Phase-summing implementation circuitry.

TABLE III

CCW SWITCHING SEQUENCE BASED ON COMPARATOR OUTPUTS

\begin{tabular}{c|c|l}
\hline \hline Drive Step & Switches to Turn On & When to Turn Switches On \\
\hline $\mathrm{XY}$ & $\mathrm{YL}$ and XU & + zero crossing of $v_{Z A C}$ \\
$\mathrm{XZ}$ & $\mathrm{XU}$ and $\mathrm{ZL}$ & - zero crossing of $v_{Z C B}$ \\
$\mathrm{YZ}$ & $\mathrm{ZL}$ and YU & + zero crossing of $v_{Z B A}$ \\
$\mathrm{YX}$ & $\mathrm{YU}$ and XL & - zero crossing of $v_{Z A C}$ \\
$\mathrm{ZX}$ & $\mathrm{XL}$ and $\mathrm{ZU}$ & + zero crossing of $v_{Z C B}$ \\
$\mathrm{ZY}$ & $\mathrm{ZU}$ and YL & - zero crossing of $v_{Z B A}$ \\
\hline \hline
\end{tabular}

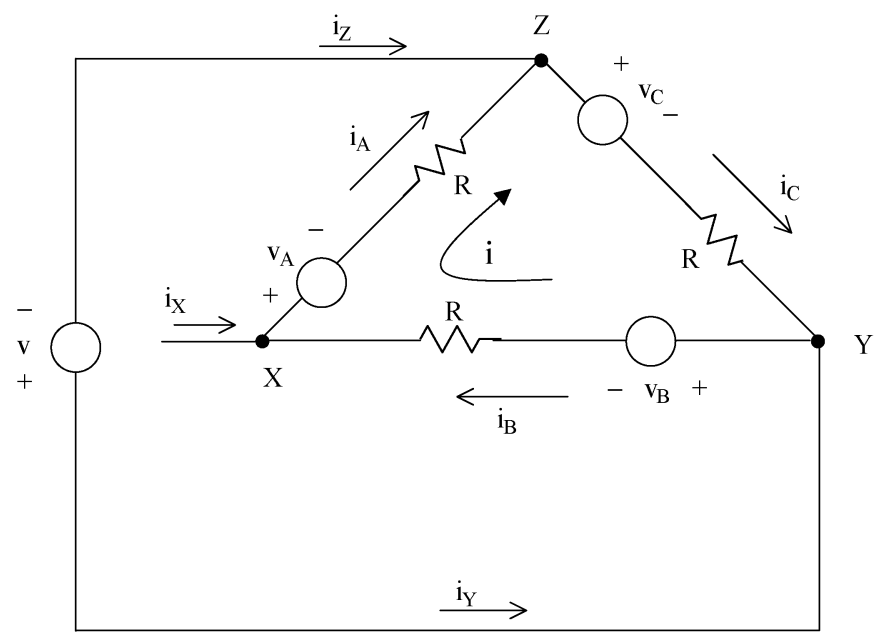

Fig. 11. Circuit model of a three-phase delta-configured motor.

\section{Delta IMPLEMENTATION}

This section discusses a geometric method of using the three node voltages for winding-sensed control for a delta-configured motor. The derivation is the dual of that shown in the preceding section, and the circuitry for delta-configured motors is the same as that for wye-configured motors. Therefore, the switch sequence logic described in this paper can be implemented for either wye-configured or delta-configured motors.

The delta-configured motor model is shown in Fig. 11 with switch sequencing in Fig. 3. We assume (as before) that the 
TABLE IV

CCW Switching Sequence For Delta-Configured Motor BASED ON INDUCED VOLTAGES

\begin{tabular}{c|c|c}
\hline \hline Drive Step & Switches to Turn On & When to Turn Switches On \\
\hline XY & YL and XU & + zero crossing of $v_{A}$ \\
XZ & XU and ZL & - zero crossing of $v_{C}$ \\
YZ & ZL and YU & + zero crossing of $v_{B}$ \\
YX & YU and XL & - zero crossing of $v_{A}$ \\
ZX & XL and ZU & + zero crossing of $v_{C}$ \\
ZY & ZU and YL & - zero crossing of $v_{B}$ \\
\hline \hline
\end{tabular}

phase windings are separated by $120^{\circ}$ electrical as shown in Fig. 4. During the third step of the switch sequence, switches YU and ZL are on. Therefore, as seen in Fig. $1, i_{Y}$ is positive and $i_{Z}$ is negative. Fig. 11 shows that this results in positive current flowing through windings $\mathrm{A}$ and $\mathrm{B}$ and negative current flowing through winding $\mathrm{C}$. Because winding $\mathrm{A}$ and $\mathrm{B}$ currents are equal, their resulting vector is equal to that of the winding $\mathrm{C}$ current (see Fig. 4). Therefore, during this $60^{\circ}$ drive step, the stator field will be pointed in the direction of the $-\mathbf{C}$ vector as shown in Fig. 4.

During the next drive step, switches YU and XL are on; $i_{Y}$ is positive and $i_{X}$ is negative. Negative current flows through windings $\mathrm{A}$ and $\mathrm{C}$ and positive current through winding $\mathrm{B}$. The stator field will then be pointed in the direction of the $\mathbf{B}$ vector shown in Fig. 4. As the switches proceed through one complete period, the stator field rotates in the $\mathrm{CCW}$ direction around the stator as shown in Fig. 4 with $60^{\circ}$ steps.

As with the wye-configured motor, we desire to maintain an average lag of $90^{\circ}$ of the rotor field with respect to the stator field. For example, since the $-\mathbf{B}$ field is at $-60^{\circ}$ in Fig. 4 , we should turn on the $-\mathbf{B}$ field when the rotor reaches $-180^{\circ}$, and turn it off when the rotor reaches $-120^{\circ}$. Likewise, we should turn on the $\mathbf{A}$ field when the rotor reaches $-120^{\circ}$, and turn it off when the rotor reaches $-60^{\circ}$.

As the permanent-magnet rotor rotates, it induces currents in the phase windings. If the motor is running at maximum torque as described earlier, then the induced voltages in the three windings will appear as the sine waves shown in Fig. 5 except they will be shifted to the right by $30^{\circ}$. The induced voltage in winding $\mathrm{B}$ lags that in winding $\mathrm{A}$ by $120^{\circ}$, and the induced voltage in winding $\mathrm{C}$ lags that in winding $\mathrm{B}$ by $120^{\circ}$. For winding-sensed control, we want to use the induced voltage zero crossings to sequence the switches. However, the only voltages that are available for sensing are the voltages at the three external nodes $X, Y$, and $Z$.

Following reasoning similar to that in Section IV, we see that we want the XY drive step to begin at the positive zero crossing of $v_{A}$. We next want the $\mathrm{XZ}$ drive step to begin $60^{\circ}$ later, which is at the negative zero crossing of $v_{C}$. The entire switch sequence is shown in Table IV.

If we can synthesize a waveform that is in phase with $v_{A}$ then we can use its positive zero crossing to turn on the XY drive step. Likewise, if can synthesize a waveform that is in phase with $\mathrm{C}$ then we can use its negative zero crossing to turn on the XZ drive step. Similar observations can be made for the other drive steps.
TABLE V

OPEN-NODE VOLTAGES FOR DELTA-CONFIGURED MOTOR

\begin{tabular}{c|c}
\hline \hline Drive Step & Open Node Voltage \\
\hline $\mathrm{XY}$ & $v_{Z}=\left(v+v_{C A}\right) / 2$ \\
$\mathrm{XZ}$ & $v_{Y}=\left(v+v_{B C}\right) / 2$ \\
$\mathrm{YZ}$ & $v_{X}=\left(v+v_{A B}\right) / 2$ \\
$\mathrm{YX}$ & $v_{Z}=\left(v+v_{C A}\right) / 2$ \\
$\mathrm{ZX}$ & $v_{Y}=\left(v+v_{B C}\right) / 2$ \\
$\mathrm{ZY}$ & $v_{X}=\left(v+v_{A B}\right) / 2$ \\
\hline \hline
\end{tabular}

Now, consider the YZ drive step. From Fig. 11,

$$
i=i_{A}=i_{B}=\frac{\left(v-v_{A}-v_{B}\right)}{2 R} .
$$

The voltage at node $X$ is given by

$$
v_{X}=v_{A}+i R
$$

Combining the two equations above results in the sensed open-node voltage

$$
v_{X}=\frac{v}{2}+\frac{v_{A B}}{2}
$$

This process can be repeated to derive the voltage at the open node for all six drive steps. The result is shown in Table V.

The virtual node is implemented as in the wye-configured case (see Fig. 8). Applying KCL at node $N_{V}$,

$$
3 v_{N V}=v_{X}+v_{Y}+v_{Z}
$$

Recognizing from Fig. 11 that $v_{Z}=0$ and $v_{Y}=v$, we obtain

$$
v_{N V}=\frac{\left(v_{X}+v\right)}{3}
$$

Combining (24) and (26), we obtain

$$
v_{N V}=\frac{v}{2}+\frac{v_{A B}}{6}
$$

Adding the circuitry shown in Fig. 9 and performing some calculations similar to those at the end of Section IV, we obtain the total comparator input voltage as

$$
v_{+}-v_{-}=-\frac{v_{C}}{2(n+1)}+\frac{(2 n-1) v_{A B}}{6(n+1)} .
$$

If we set $n=2$ in this equation we obtain

$$
v_{+}-v_{-}=\frac{\left(-v_{C}+v_{A B}\right)}{6}
$$

Combining $v_{A}+v_{B}+v_{C}=0$ (from Figs. 5 and 7),

$$
v_{+}-v_{-}=\frac{v_{A}}{3}
$$

Therefore, the negative zero crossing of the comparator output $\left(v_{+}-v_{-}\right)$of Fig. 9 is the same as the negative zero crossing of $v_{A}$. However, it is exactly at this point that we want to switch from the present $\mathrm{YZ}$ drive step to the next drive step (see Table IV). Therefore, the negative zero crossing of $\left(v_{+}-v_{-}\right)$ can be used to begin the YX drive step. 


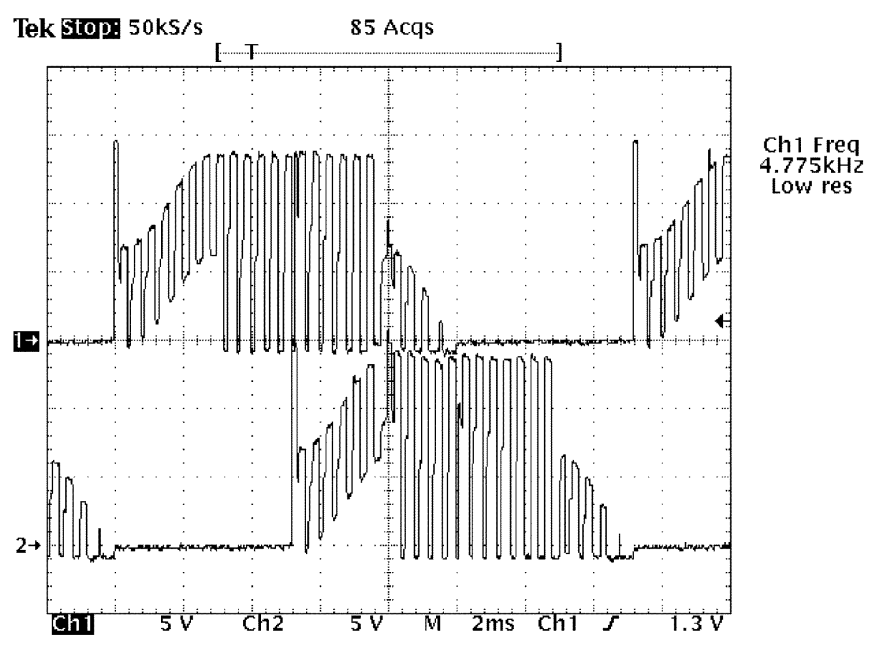

Fig. 12. Experimental results for a delta-configured motor.

Another major advantage of vector-summed control is the common-mode rejection of pulsewidth-modulation (PWM) noise (due to $v$ ) at the switching time. Note that (30) is not a function of $v$. Consequently, this scheme works well at low motor speeds.

This reasoning can be extended to derive the switch sequencing for the complete switching pattern. Both circuitry and switch sequencing is the same as that for the wye-configured motor, as shown in Table III. The winding-sensed switching presented in this paper can thus be implemented regardless of whether the motor is wye or delta configured.

\section{EXPERIMENTAL RESULTS}

Vector-summed commutation has been tested with both winding configurations. The results for a delta-configured motor are given here. The waveforms at two motor terminals are shown in Fig. 12, with trace 1 leading trace 2 by $120^{\circ}$. The driver PWM frequency was about $3 \mathrm{kHz}$ and the motor electrical frequency was about $67 \mathrm{~Hz}$.

While either terminal is open, the induced-voltage vector sum of the phase-windings connected to it results in an induced-voltage waveform, with the PWM drive waveform superimposed upon it. In trace 1, the rising waveform shape is evident from the beginning of the second division through the first half of the third division. Around the peaks of each half-cycle, the terminal is driven. This is seen in trace 1 from the last half of the third division through the fifth division. During the drive interval, the full amplitude of the PWM waveform appears, and is flat on top, at the applied source voltage of $v$. When not driven, the PWM amplitude is about half of the drive voltage.

Notice that the drive intervals for both waveforms are centered around the peaks, indicating good phase alignment. This centering remained constant over a wide motor speed range and under mild to no-load testing.

When we add an open-terminal induced voltage (for example, $v_{X}$ ) to one of the driven terminal voltages (for example, $v_{Y}$ ), we obtain a sum that is not equal to the induced voltage sum
$v_{A}+v_{B}$. Consequently, the desired vector, $v_{A}+v_{B} / 2$, is not the constructed vector using this additive implementation. However, the only angle at which the reconstruction must be correct is at the switching angle, and it is only at this angle that the construction is indeed correct, and the induced voltage equals the terminal voltage. Consequently, the comparator switches at the right angle.

One of the observed characteristics of this scheme is the common-mode rejection of the switching noise at the comparator inputs. The PWM noise on the waveform decreases as the switching angle is approached from either side. This is a result of the constructed voltage approaching the desired addition of the induced-voltage vectors. In one implementation of this scheme, the mere vibration of the motor shaft was sufficient to start the motor with no observed misbehavior due to incorrect phase control. That is, the comparator noise was sufficiently rejected at low speed to correctly sense the added induced-voltage waveform zero crossings.

Similar results were obtained with the wye configuration. While the motor was running, the neutral-node connection was switched between the physical motor neutral terminal and a virtual neutral node, synthesized as shown in Fig. 9. No difference in behavior was observed, electrically or mechanically.

\section{CONCLUSION AND DISCUSSION}

We have presented a simple but elegant method for deriving the switching sequence of a winding-sensed motor drive for three-phase PMS motors. The method works for both wye- and delta-configured motors and results from a vector-summing approach to induced voltages. The implementation is low in cost as it requires only nine resistors and three comparators. The winding-sensed drive described in this paper can easily be implemented without a microcontroller or digital signal processor. However, some firmware may be desired for a more refined implementation that includes phase-winding impedance compensation, startup logic, and control interfacing. The experimental results show good phase control of the vector-summed method.

\section{REFERENCES}

[1] R. Becerra, T. Jahns, and M. Ehsani, "Four quadrant sensorless brushless ECM drive," in Proc. IEEE APEC'91, 1991, pp. 202-209.

[2] H. Chen and C. Liaw, "Sensorless control via intelligent commutation tuning for brushless DC motor," Proc. IEE-Elect. Power Applicat., vol. 146, no. 6, pp. 678-684, Nov. 1999.

[3] Z. Chen, M. Tomita, S. Doki, and S. Okuma, "New adaptive sliding observers for position- and velocity-sensorless controls of brushless DC motors," IEEE Trans. Ind. Electron., vol. 47, pp. 582-591, June 2000.

[4] A. Consoli, G. Scarcella, and A. Testa, "Industry application of zerospeed sensorless control techniques for PM synchronous motors," IEEE Trans. Ind. Applicat., vol. 37, pp. 513-521, Mar./Apr. 2001.

[5] T. Hsien, Y. Sun, and M. Tsai, " $\mathrm{H}^{\infty}$ control for a sensorless permanent-magnet synchronous drive," Proc. IEE-Elect. Power Applicat., vol. 144, no. 3, pp. 173-181, May 1997.

[6] D. Jung and I. Ha, "Low-cost sensorless control of brushless DC motors using a frequency-independent phase shifter," IEEE Trans. Power Electron., vol. 15, pp. 744-752, July 2000.

[7] N. Kasa and H. Watanabe, "A mechanical sensorless control system for salient-pole brushless DC motor with autocalibration of estimated position angles," IEEE Trans. Ind. Electron., vol. 47, pp. 389-395, Apr. 2000. 
[8] S. Lau, J. Gardner, and D. Beselowitz, "Sensorless position control of a brushless motor in circulatory assist devices," Mechatronics, vol. 1, no. 3, pp. $277-292,1991$.

[9] F. Lin, K. Shyu, and R. Wai, "DSP-based minmax speed sensorless induction motor drive with sliding mode model-following speed controller," Proc. IEE-Elect. Power Applicat., vol. 146, no. 5, pp. 471-478, Sept. 1999.

[10] S. Ogasaware and H. Akagi, "Implementation and position control performance of a position-sensorless IPM motor drive system based on magnetic saliency," IEEE Trans. Ind. Applicat., vol. 34, pp. 806-812, July/Aug. 1998.

[11] S. Ostlund and M. Brokemper, "Sensorless rotor-position detection from zero to rated speed for an integrated PM synchronous motor," IEEE Trans. Ind. Applicat., vol. 32, pp. 1158-1165, Sept./Oct. 1996.

[12] K. Rajashekara and A. Kawamura, Sensorless Control of AC Motor Drives. New York: IEEE Press, 1996.

[13] M. Schroedl, "Sensorless control of permanent magnet synchronous motors," Elect. Mach. Power Syst., vol. 22, pp. 173-185, 1994.

[14] T. Song, M. Rahman, K. Lim, and M. Rahman, "A singular perturbation approach to sensorless control of a permanent magnet synchronous motor drive," IEEE Trans. Energy Conversion, vol. 14, pp. 1359-1365, Dec. 1999.

[15] K. Tatematsu, D. Hamada, K. Uchida, S. Wakao, and T. Onuki, "New approaches with sensorless drives," IEEE Ind. Applicat. Mag., vol. 6, pp. 44-50, July/Aug. 2000.

[16] C. Tsai and H. Lu, "Observer-based speed estimation method for sensorless vector control using artificial neural network," Elect. Mach. Power Syst., vol. 28, pp. 861-873, 2000. 